\title{
User Scheduling for Coordinated Dual Satellite Systems with Linear Precoding
}

\author{
Dimitrios Christopoulos*, Symeon Chatzinotas* and Björn Ottersten* \\ ${ }^{*} \mathrm{SnT}$ - securityandtrust.lu, University of Luxembourg \\ email: \{dimitrios.christopoulos, symeon.chatzinotas, bjorn.ottersten\}@uni.lu
}

\begin{abstract}
The constantly increasing demand for interactive broadband satellite communications is driving current research towards novel system architectures that reuse frequency in a more aggressive manner. To this end, the topic of dual satellite systems, in which satellites share spatial (i.e. same coverage area) and spectral (i.e. full frequency reuse) degrees of freedom is introduced. In each multibeam satellite, multiuser interferences are mitigated by employing zero forcing precoding with realistic per antenna power constraints. However, the two sets of users that the transmitters are separately serving, interfere. The present contribution, proposes the partial cooperation, herein referred to as coordination, between the two coexisting transmitters in order to reduce interferences and enhance the performance of the whole system, while maintaining moderate system complexity. In this direction, a heuristic, iterative, low complexity algorithm that allocates users in the two interfering sets is proposed. This novel algorithm, improves the performance of each satellite and of the overall system, simultaneously. The first is achieved by maximizing the orthogonality between users allocated in the same set, hence optimizing the zero forcing performance, whilst the second by minimizing the level of interferences between the two sets. Simulation results show that the proposed method, compared to conventional techniques, significantly increases spectral efficiency.
\end{abstract}

\section{INTRODUCTION}

Towards the next generation of broadband multibeam Satellite Communication (SatCom) systems, innovative system architectures need to be considered in order to meet the highly increasing demand for throughput and close the digital divide. Spectrum scarcity is a major obstacle, especially in a satellite context where the higher frequency bands exhibit challenging channel impairments. In this direction, the investigation of full frequency reuse techniques that exploit the spatial degrees of freedom offered by the multibeam antenna is necessitated. Furthermore, in the evolution of geostationary (GEO) satellite systems, orbital slot congestion is an uprising problem. The fact that unpredictable changes in the traffic demand might cause the launch of secondary satellites that support existing ones should be considered as well. More importantly, long periods of coexisting satellites appear during the replacement phase of old satellites. Finally, cooperation between multibeam satellites has the potential of overcoming the major issue of Adjacent Satellite Interferences (ASI). All the above arguments provide reasonable cause towards the investigation of the optimal coexistence of two satellites.
Existing literature on Multiuser Multiple Input Multiple Output (MU-MIMO) antennas, provides transmitter techniques to alleviate the multiuser interferences in many cases [2]. Furthermore, linear low complexity techniques have proven more realistic in terms of practical implementation. Specifically, focusing on the forward link (FL) of multibeam SatComs, linear joint processing techniques have shown great potential by providing a substantial tradeoff between implementation complexity and near optimal performance in terms of SumRate (SR) [3]. Specifically, Zero Forcing (ZF) precoding, in detail analyzed further on, is based on channel inversion and then optimal power allocation over the users, with the aim of maximizing some performance metric. The metrics commonly addressed in literature involve either the total throughput performance (i.e. max SR criterion) or the Signal to Interference plus Noise Ratio (SINR) level of the worst user (i.e. max fairness criterion). Another important parameter in linear precoding is the type of constraints that will be assumed. Usually, a total sum power constraint simplifies the analysis and provides better results since the available power is freely allocated in every antenna. Despite its performance, the sum power constraint is unrealistic since each satellite antenna is fed by a dedicated high power amplifier (HPA) operating close to saturation. Hence, power cannot be allocated freely amongst the transmit antennas and a per antenna power constraint should be considered. In the present work, each satellite employs ZF beamforming while the power allocation is optimized under max SR criteria, subject to realistic per antenna constraints.

Albeit the aforementioned technique, in a dual satellite system, intersatellite interferences still need to be handled. To completely mitigate these interferences, without frequency orthogonalization, full cooperation between the two transmitters has to be employed. Subsequently, the cooperating multi-antenna transmitters should perform joint or coherent transmission to all users while, both data and channel state information (CSI) must be exchanged [4]. In a SatCom context, each satellite is served by one or multiple dedicated gateways, thus a fully cooperative dual satellite system would require a large number of interconnected GWs that exchange a substantial load of information. In the light of the above observations, partial cooperation (i.e. coordination) between the two satellites is proposed, therefore reducing the amount 
of data exchanged.

The rest of the present paper is structured as follows. A brief review of the existing related work is provided in Section II. The considered system model is described with detail in Section III. Section IV explores the theoretical aspects of the developed heuristic algorithm, explaining the intuition behind it. In Section V, simulation results for the performance of the proposed algorithm are presented. Conclusions are drawn in Section VI, along with future extensions of the present work.

\section{RELATED WORK}

Linear precoding techniques and especially ZF have been extensively investigated for terrestrial systems in [5], [6] and the references therein. Despite their suboptimality, these techniques can still achieve asymptotically optimal performance under specific conditions, as proven in [6]-[8]. Specifically, if user channels are perfectly orthogonal to each other, ZF will attain maximum performance. Under the assumption of large random user sets, the probability of orthogonal users increases. User selection, however, is a highly complex problem. Despite its complexity, simple suboptimal algorithms in the existing literature provide substantial gains with affordable complexity. Based on existing algorithms, Yoo et al [7] proposed an iterative user selection algorithm that allows ZF to achieve the performance of non-linear precoding [9] when the number of available for selection users grows to infinity.

Despite the extensive literature on linear precoding and user selection, herein we further consider the optimal allocation of the selected users in two coexisting groups that interfere with each other. The novelty of the proposed work lies in the fact that user selection and allocation, not only optimizes the $\mathrm{ZF}$ performance of each system but also considers the interaction between the two transmitters, i.e. the inter-satellite interference. The procedure of selecting users out of a large pool and allocating them to specific sets is referred to as user scheduling.

Notation: Throughout the paper, $\mathcal{E}[\cdot],(\cdot)^{\dagger},\|\cdot\|$ denote the expectation, the conjugate transpose and the norm operations, respectively. Bold face lower case characters denote column vectors and upper case denote matrices while $\mathbf{I}_{n}$ denotes an identity matrix of size $n$. Upper case calligraphic characters denote sets. Operations $\mathcal{A}=\emptyset$, and $\mathcal{A}-\mathcal{B}$ define an empty set and the relative complement of $\mathcal{B}$ in $\mathcal{A}$, respectively. Finally $|\mathcal{A}|$, denotes the cardinality of a set.

\section{SySTEM MODEL}

The system under investigation consists of two collocated multibeam satellites with overlapping coverage areas, serving fixed single antenna users (Fixed Satellite Services, FSS). A large number of users uniformly distributed in each beam is assumed and a Time Division Multiple Access (TDMA) scheme is realized, leading to one user per beam served, during each time slot. An overview of the considered system is depicted in Fig. 1, where the focus is on the Forward Link (FL) downlink of the satellites (i.e. the link between the satellite and the users), while the FL uplink, or feeder

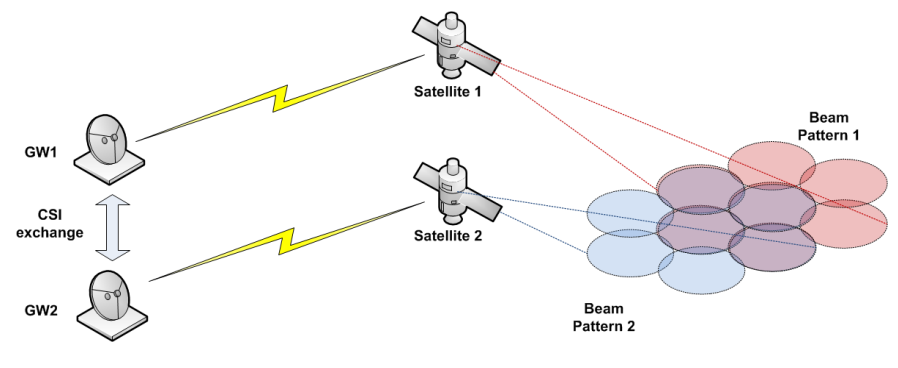

Fig. 1. System model

link (i.e. the link between each earth gateway station and the corresponding satellite), is considered ideal. It should be clarified that for simplicity purposes the assumed model does not account for the earth curvature and the satellite orbit geometry. Subsequently, the variations in the distances of the beam centers and the distance between the satellites are not modeled but will be handled in future extensions.

Considering each multibeam satellite separately, linear precoding is employed to cancel out multiuser interferences. By denoting $N$ and $K$ the number of transmit antennas and single antenna users respectively, the one user per beam per timeslot assumption implies a symmetric system, i.e. $N=K$. Subsequently, in each separate transmitter, a Multi-User (MU) Multiple Input Single Output (MISO) Broadcast Channel (BC) is realized and the input-output analytical expression for the $k$-th user reads as

$$
y_{k}=\mathbf{h}_{k}^{\dagger} \mathbf{x}+n_{k}
$$

where $\mathbf{h}_{k}^{\dagger}$ is an $1 \times N$ vector composed of the channel coefficients between the $k$-th user and the $N$ antennas (i.e. feeds) of the satellite, $\mathrm{x}$ is an $N \times 1$ vector of transmitted symbols and $n_{k}$ is the independent identically distributed (i.i.d) zero mean Additive White Gaussian Noise (AWGN) measured at the $k$-th user's receive antenna. The noise is assumed normalized, thus $\mathcal{E}\left\{\left|n_{k}\right|^{2}\right\}=1$.

To accurately model the multibeam satellite channel the following considerations are made. Under the assumption of fixed users with highly directive antennas, the satellite channel can be modeled as AWGN channel with real channel gains ${ }^{1}$ that depend only on the multibeam antenna pattern and on the user position. The elements of the $k$-th user's channel vector are the square roots of the gain coefficients calculated using the well accepted method of Bessel functions [10]

$$
g_{i k}\left(\theta_{i k}\right)=G_{\max }\left(\frac{J_{1}(u)}{2 u}+36 \frac{J_{3}(u)}{u^{3}}\right)^{2},
$$

where $u=2.07123 \sin \theta / \sin \theta_{3 d B}, J_{1}, J_{3}$ are the Bessel functions of the first kind, of order one and three respectively and $G_{\max }$ is the maximum axis gain of each antenna. The $k$-th users' position corresponds to an off-axis angle $\theta_{i k}$ with respect to the boresight of the $i$-th beam where $\theta_{i}=0^{\circ}$.

\footnotetext{
${ }^{1}$ The main conclusions of this study, still apply if a random phase is incorporated in the channel gains.
} 


\section{A. Transmit beamforming}

Transmit beamforming is a multiuser precoding technique that separates user data streams in different beamforming directions [6]. Let us denote $s_{k}, \mathbf{w}_{k}$ and $p_{k}$ as the unit power symbol, the $N \times 1$ transmit precoding vector and the power scaling factor respectively, corresponding to the $k$-th user. The total transmit signal will then read as

$$
\mathbf{x}=\sum_{k=1}^{K} \sqrt{p_{k}} \mathbf{w}_{k} s_{k}
$$

Subsequently, when beamforming is employed, (1) will become

$$
y_{k}=\mathbf{h}_{k}^{\dagger} \sqrt{p_{k}} \mathbf{w}_{k} s_{k}+\mathbf{h}_{k}^{\dagger} \sum_{j \neq k} \sqrt{p_{j}} \mathbf{w}_{j} s_{j}+n_{k},
$$

where the first term of the summation refers to the useful signal and the second to the interferences. The $N \times 1$ vector $\mathbf{w}_{k}$ is the $k$-th user's precoding vector, that is the $k$-th column of a total precoding matrix $\mathbf{W}=\left[\mathbf{w}_{1}, \mathbf{w}_{2}, \ldots, \mathbf{w}_{k}\right]$. Subsequently, the SINR at each reads as

$$
\operatorname{SINR}_{k}=\frac{p_{k}\left|\mathbf{h}_{k}^{\dagger} \mathbf{w}_{k}\right|^{2}}{1+\sum_{j \neq k} p_{j}\left|\mathbf{h}_{k}^{\dagger} \mathbf{w}_{j}\right|^{2}} .
$$

When beamforming is employed, determining the optimal precoding vectors and power allocation vectors is tedious in practice. In the following paragraph a very common practical scheme, that will be employed in this paper is presented.

\section{B. Zero Forcing beamforming}

The implementation complexity of the MIMO BC channel capacity achieving dirty paper coding [9], led to the development of less complex, yet suboptimal techniques. A linear precoding technique with reasonable computational complexity that still achieves full spatial multiplexing and multiuser diversity gains, is ZF precoding [6], [11], [12]. The ability of $\mathrm{ZF}$ to fully cancel out multiuser interference makes it useful for the high SNR regime. However, it performs far from optimal in the noise limited regime. Also it can only simultaneously serve at most equal to the number of transmit antennas, single antenna users. A common solution for the ZF precoding matrix is the pseudoinverse of the $K \times N$ channel matrix $\mathbf{H}=\left[\mathbf{h}_{1}, \mathbf{h}_{2} \ldots, \mathbf{h}_{k}\right]^{\dagger}$. Under a total power constraint, channel inversion is the optimal precoder choice in terms of maximum SR and maximum fairness [5]. However, according to the same authors, when Per Antenna power Constraints (PAC) are assumed, optimization over the parameters of a generalized inverse has to be performed. For simplicity, the present work only considers the simplified channel inversion design. Finally, due to the symmetrical system, the precoding matrix will read as $\mathbf{W}=\mathbf{H}^{-1}$.

Following the complete mitigation of interferences, the SINR at the $k$-th user will read as $\operatorname{SINR}_{k}^{Z F}=p_{k}\left|\mathbf{h}_{k}^{\dagger} \mathbf{w}_{k}\right|^{2}$. Subsequently, the problem reduces to a power allocation optimization process, where the aim is to maximize the total system SR, subject to per antenna power constraints. This problem is formalized as

$$
\begin{aligned}
\max _{\left\{p_{k}\right\}} & \sum_{k=1}^{K} \log _{2}\left(1+\mathrm{SINR}_{k}^{Z F}\right), \\
\text { s.t. } & \sum_{k=1}^{K} p_{k} \mathbf{w}_{k}^{\dagger} \mathbf{Q}_{j j} \mathbf{w}_{k} \leq P_{j}, j=1, \cdots, K .
\end{aligned}
$$

where $\mathbf{Q}_{j j}$ is an $K \times K$ zero matrix, with the $j j$-th element equal to one and $P_{j}=P_{t o t} / K$, for every $j$, since we assume that all satellite antennas have identical RF chains. Problem (6), is a standard convex optimization problem [13], [14] that is solved using common convex optimization tools [15].

\section{Dual satellite systems: channel model}

Extending the previous considerations to a dual satellite scenario, the resulting channel needs to be modeled. In this direction, two overlapping clusters of $N_{1}$ and $N_{2}$ spot-beams covering $K_{1}$ and $K_{2}$ fixed user terminals respectively, are considered (Fig. 1) . The users, each equipped with a single antenna, are uniformly distributed over the coverage area. Despite the fact that in each satellite separately, a MU MISO $\mathrm{BC}$ is realized, the whole system operates over an interference channel. The $k$-th user now has two vector channels, one towards each satellite, denoted using the indices 1 and 2 respectively. The channel vectors $\mathbf{h}_{k 1}^{\dagger}, \mathbf{h}_{k 2}^{\dagger}$ are the rows of a total channel matrix $\mathbf{H}_{t o t}$ of $\left(K_{1}+K_{2}\right) \times\left(N_{1}+N_{2}\right)$ dimensions, that models the satellite antenna gains of the two satellites. Assuming that the $k$-th user is allocated to the first satellite its SINR will read as

$$
\mathrm{SINR}_{k}=\frac{p_{1 k}\left|\mathbf{h}_{k 1}^{\dagger} \mathbf{w}_{k 1}\right|^{2}}{1+\sum_{j=1}^{K_{2}} p_{2 j}\left|\mathbf{h}_{k 2}^{\dagger} \mathbf{w}_{j 2}\right|^{2}} .
$$

In (7), the interferences from the adjacent satellite are clearly noted, while inter-satellite multiuser interferences are completely mitigated by the the precoding. The equivalent relations for the users allocated to the second satellite, are straightforward to deduce, by exchanging indices 1 and 2 .

\section{User Selection And Allocation}

As proven in [7], user selection can significantly improve the performance of $\mathrm{ZF}$ in an individual system. However, considering the coexistence of two separate transmitters, as is the case in a dual satellite system, partial cooperation, namely coordination, can be employed to solve the problem of high intersatellite interferences. It should also be noted that current research in cognitive SatComs is considering scenarios where two satellites coexist (e.g. [1]). In the same direction, this contribution proposes an algorithm that selects users and allocates them to each satellite. Intuitively, the two basic criteria that need to be considered for this procedure are: a) the maximization of the performance of each satellite separately, and b) the minimization of the interferences between the two sets.

The performance of each satellite separately is optimized by constructing a semi-orthogonal user group from a vast number 
of users [6]. Extending this result, the creation of two user sets under the semi-orthogonality criterion is straightforward since the channel gain of each user can be projected to the orthogonal complement of the channels of the previously selected users. In each iteration of the algorithm, the user with the maximum projection is allocated to the corresponding set. This simplistic approach has been considered for comparison reasons.

The novel proposed algorithm accounts for the effects of the interferences between the two sets. It should first be mentioned that the exact calculation of the level of interferences in each iteration is not possible since the exact user set is still undetermined. To exactly calculate the interferences, one would need to solve the power optimization problem (6) for all possible combinations of users. Under the assumption of large number of users, this would lead to unaffordable computational complexity. However, based on a basic advantage of $\mathrm{ZF}$ beamforming, which is the decoupled nature of the precoder design and the power allocation optimization problems, an approximation of the interferences can be made. In this direction, the precoding vectors of the users selected in the previous iterations can be utilized to provide an indicative measure of the interferences between the user sets. This implies that an equal power allocation is assumed. This assumption becomes asymptotically exact in the high SNR regime, where the powers allocated to each user are approximately equal. Incorporating all the above, a heuristic, iterative Semi-orthogonal Interference aware User Allocation algorithm (SIUA) has been developed and will be presented in the following.

The SIUA algorithm, presented in full detail in Algo. 1 works as follows. During the initialization procedure, i.e. Step 1 , the strongest user towards each satellite is allocated to the equivalent group. While the two sets are not full, Steps 2 and 3 are executed. In Step 2, for each of the unallocated users, the following metrics are calculated: a) In accordance to [6], $\mathbf{g}_{1 k}$ and $\mathbf{g}_{2 k}$ represent the orthogonal component of each unallocated user's channel to the orthogonal subspace of the already allocated users, for the two sets respectively. In b) $\mathrm{I}_{k 1}^{r}$ and $\mathrm{I}_{k 2}^{r}$ are equivalent measures for the interference each user would receive if equal power allocation is employed. It is calculated as the squared norm of the product of the users' channel with the power of the transmit signal of the second user set and the channel of each user. Finally, in c) $\mathrm{I}_{k 1}^{i}$ and $I_{k 2}^{i}$ are approximations of the interference that the allocation of each user can potentially induce to the second set, if this user is allocated in the respective set. It is calculated as the product of the interferences this user induces to every user that belongs to the second set. Since the goal is to find the most orthogonal users that at the same time receive and induce the least possible interferences, the measure to be maximized is the fraction of the orthogonality metric over the product of the interference metrics. At the last stage of each iteration, two maximum fractions $\mu_{1}$ and $\mu_{2}$ are calculated over the total user set and compared between them. The user that corresponds to the largest measure among the two, is allocated to the
TABLE I

LINK BUDGET PARAMETERS

\begin{tabular}{l|c} 
Parameter & Value \\
\hline Orbit & GEO \\
Frequency band & Ka $(20 \mathrm{GHz})$ \\
User link bandwidth & $500 \mathrm{MHz}$ \\
Number of beams & 7 \\
Beam diameter & $600 \mathrm{Km}$ \\
\hline TWTA RF power @ saturation & $+21 \mathrm{dBW}$ \\
Max satellite antenna gain $G_{T}$ & $+52 \mathrm{dBi}$ \\
Max user antenna Gain $G_{R}$ & $+40 \mathrm{dBi}$ \\
Free space loss & $-210 \mathrm{~dB}$ \\
\hline Signal Power S & $-97 \mathrm{dBW}$ \\
\hline Receiver noise power $N$ & $-118 \mathrm{dBW}$ \\
\hline SNR S/N & $21 \mathrm{~dB}$ \\
\hline
\end{tabular}

equivalent satellite.

The described heuristic and iterative optimization algorithm, requires full knowledge of the CSI of all users. Consequently, coordination reduces the amount of data that needs to be exchanged since each GW handles only the data of the users allocated to the corresponding satellite. Moreover, SIUA runs only as many times as the number of transmit antennas and thus comprises a scalable solution that can be extended for larger multibeam systems. Another advantage of this solution is that the power optimization in each satellite, a convex optimization problem that requires some computational complexity, is decoupled from the algorithm execution. Additionally, despite the fact that the solution is heuristic and not optimal, it is considerably less complex since the optimal user allocation would require exhaustive search of all possible combinations of the users. Finally, SIUA, can be executed in a centralized location or run in parallel at the GWs that share CSI.

\section{Simulation RESUlts}

To evaluate the performance of the proposed algorithm, two satellites each with seven beams where assumed. The low number of beams is only chosen to reduce the simulation time of the convex optimization problem (6) and has no effect on the algorithm, as discussed in Sec. IV. It is however inline with the future considerations for the terabit satellites, where each GW is expected to handle between 5 and 8 beams. Additionally, the simulations are performed according to the link budget calculations described in Table I, where it can be noted that the normal SNR operating point of current satellite systems is $21 \mathrm{~dB}$.

In Fig. 2, the results of Monte Carlo simulations that calculate the performance in terms of system SR -given by (6)- of the coexisting systems with and without cooperation, are presented. Due to random user positioning, 100 iterations where executed, each with a different user position pattern so that the average performance can be evaluated. The upper bound for the system performance is deduced assuming full cooperation amongst the transmitters. To be more precise, this is equivalent to one large satellite performing linear precoding over a channel matrix $\mathbf{H}_{t o t}$ of $\left(K_{1}+K_{2}\right) \times\left(N_{1}+N_{2}\right)$ dimensions. For this case two curves show the SR in bps/Hz: one for the average performance of random user positioning 
and one employing the algorithm developed in [6], namely the Semi-orthogonal User allocation (SUS) algorithm, which allocates users without regarding the coexistence of the systems. Subsequently, an average gain of $25 \%$ is noted by employing a simple user selection scheme, instead of randomly selecting users. Since in the fully cooperative system, interferences are completely mitigated, SIUA is unnecessary.

The substantial performance gain from SIUA is proven in Fig. 2 for the more realistic case of coordinated systems. In this figure, SIUA is compared to SUS and also to an independent interfering system. From these curves, it is concluded that in the low SNR region, the SUS algorithm performs better, as expected since the noise limited regime is almost interference free. In the SNR region of interest, however, it is clear that the SIUA algorithm, by reducing the level of interferences, provides substantial gains: more than $52 \%$ of improvement in terms of SR, over a non cooperative system and $28 \%$ of improvement over a coordinated system employing simple user selection. It is therefore concluded that the SIUA sacrifices some low SNR performance to provide substantial gains in the SNR region of interest. A simple switching scheme between the two algorithms can provide good performance over the whole SNR region of interest.

In Fig. 3, a coordinated system using SIUA, is compared to an ideal non interfering dual satellite system that employs frequency orthogonalization to allow the operability of the two coexisting satellites. This approach models the currently employed techniques of bandwidth splitting. In this plot it is proven that around the SNR area of interest, the proposed algorithm outperforms the conventional techniques (25\% gain). Therefore, the SIUA comprises a candidate tool for handling ASI. As the SNR increases, the gain decreases as expected, since the conventional system operates under the ideal assumption of zero interferences.

Finally, in Fig. 4, the behavior of the algorithm with respect to the size of the user pool is investigated. To this end, the achievable SR for a given value of SNR, i.e. $20 \mathrm{~dB}$, is calculated as the total number users increases and also compared to the performance of the SUS algorithm. In this figure it is proven that the algorithm reaches close to its maximum performance for a finite number of total users $(600$ users) and further increase of the user pool has little effects. From the same figure we note that the rate of convergence of the proposed technique is very similar to the SUS algorithm.

\section{CONCLUSIONS AND FUTURE WORK}

The topic of dual satellite systems is addressed in the present contribution and a suboptimal, simple solution is proposed. A low complexity, heuristic algorithm that minimizes the interferences between the two groups, while maintaining the orthogonality between the users of the same group, allows for the coexistence of two separate multibeam, joint processing, coordinated satellites. Thereby the overall system spectral efficiency is increased. The only cost of the proposed solution is the CSI exchange between the GWs. According to simulation results, the algorithm achieves $52 \%$ of spectral

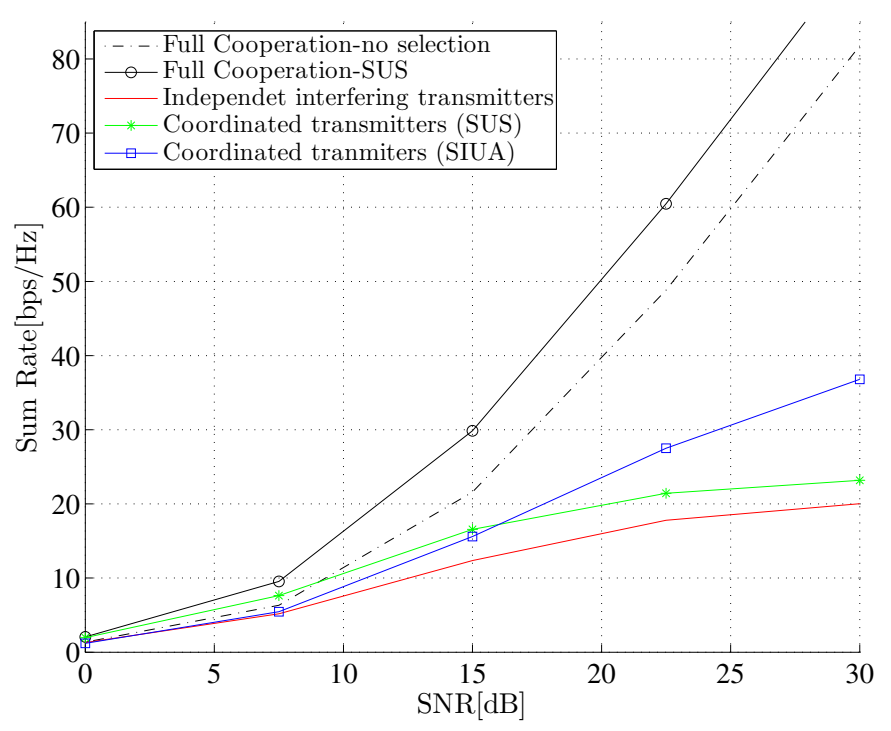

Fig. 2. Evaluation of SIUA algorithm in terms of system sum rate, by comparison with optimal and interfering systems.

efficiency improvement over non-cooperative full frequency reuse systems and $25 \%$ improvement over non-cooperative conventional orthogonalized in the frequency domain systems. Subsequently, the proposed scheme, successfully exploits the spatial orthogonalization of users and allows for systems that are partially cooperative to operate over all the available spectrum.

This fundamental approach can be extended as a useful interference mitigation technique for several other scenarios. Also, the consideration of a third set of users that will be simultaneously served by both satellites to achieve some minimum requirements will be part of future work. The dual satellite system will also be investigated as a cognitive communications system where priority will be given to primary users.

\section{ACKNOWLEDGMENT}

This work was partially supported by the National Research Fund, Luxembourg under the project " $C O{ }^{2} S A T$ : Cooperative \& Cognitive Architectures for Satellite Networks' and by the seventh framework program project " $C O R A S A T$ : COgnitive RAdio for SATellite Communications.

\section{REFERENCES}

[1] M. Costa, "Writing on dirty paper," IEEE Trans. Inf. Theory, vol. 29, no. 3, pp. 439-441, 1983.

[2] D. Christopoulos, S. Chatzinotas, G. Zheng, J. Grotz, and B. Ottersten, "Linear and non-linear techniques for multibeam joint processing in satellite communications," EURASIP J. Wirel. Commun. Netw., no. 162, 2012. [Online]. Available: http://jwcn.eurasipjournals.com/content/ 2012/1/162

[3] Y. Huang, G. Zheng, M. Bengtsson, K.-K. Wong, L. Yang, and B. Ottersten, "Distributed multicell beamforming with limited intercell coordination," IEEE Trans. Signal Process., vol. 59, no. 2, pp. $728-738$, feb. 2011.

[4] A. Wiesel, Y. C. Eldar, and S. Shamai, "Zero forcing precoding and generalized inverses," IEEE Trans. Signal Process., vol. 56, no. 9, pp. 4409-4418, Sept. 2008. 


\section{SIUA algorithm}

Output: $\mathcal{S}_{1} \& \mathcal{S}_{2}$

Step 1: $\forall k=1,2 \ldots M$ allocate the strongest channel norm to each satellite:

$\pi_{1(1)}=\arg \max \left\|\mathbf{h}_{k 1}\right\|, \mathbf{g}_{1(1)}=\mathbf{h}_{\pi_{1} 1}$

$\pi_{2(1)}=\arg \max \left\|\mathbf{h}_{k 2}\right\|, \quad \mathbf{g}_{2(1)}=\mathbf{h}_{\pi_{2} 2}$

$\mathcal{S}_{1}=\pi_{1(1)}, \mathcal{S}_{2}=\pi_{2(1)}$

$\mathcal{T}=\{1, \ldots M\}-\left\{\pi_{1(1)}, \pi_{2(1)}\right\}$ set of unprocessed users

$i=1$ iteration counter

while $\left(\left|\mathcal{S}_{1}\right|<M_{1}\right) \&\left(\left|\mathcal{S}_{2}\right|<M_{2}\right)$

do

Step 2: forall elements of $\mathcal{T}_{(i)}$ do

(a) $\mathbf{g}_{1 k}=\mathbf{h}_{1 k}\left(\mathbf{I}_{K}-\sum_{j=1}^{i-1} \frac{\mathbf{g}_{1(j)}^{\dagger} \mathbf{g}_{1(j)}}{\| \mathbf{g}_{1(j) \|^{2}}}\right)$

$\mathbf{g}_{2 k}=\mathbf{h}_{k 2}\left(\mathbf{I}_{K}-\sum_{j=1}^{i-1} \frac{\mathbf{g}_{2(j)}^{\dagger} \mathbf{g}_{2(j)}}{\| \mathbf{g}_{2(j) \|^{2}}}\right)$

(b) $\mathrm{I}_{1 k}^{r}=\mathbf{h}_{k 2}\left(\mathbf{W}_{2} \mathbf{W}_{2}^{\dagger}\right) \mathbf{h}_{k 2}^{\dagger}$

$\mathrm{I}_{2 k}^{r}=\mathbf{h}_{k 1}\left(\mathbf{W}_{1} \mathbf{W}_{1}^{\dagger}\right) \mathbf{h}_{k 1}^{\dagger}$

(c) $\mathrm{I}_{1 k}^{i}=\prod_{l \epsilon \mathbf{t}}^{l \neq k}\left(\mathbf{h}_{l 1}\left(\mathbf{W}_{1 k} \mathbf{W}_{1 k}^{\dagger}\right) \mathbf{h}_{l 1}^{\dagger}\right)$

$\mathrm{I}_{2 k}^{i}=\prod_{l \epsilon \mathbf{t}}^{l \neq k}\left(\mathbf{h}_{l 2}\left(\mathbf{W}_{2 k} \mathbf{W}_{2 k}^{\dagger}\right) \mathbf{h}_{l 2}^{\dagger}\right)$

where $\mathbf{W}_{n}, n=1,2$ is the $\mathrm{ZF}$ precoding matrix of each satellite with users allocated from previous iterations and $\mathbf{W}_{n k}, k \epsilon \mathbf{t}$ is the same end matrix but with the $k$ th user added.

Step 3: $\mu_{1(i)}=\max \left\{\left\|g_{1 k}\right\| /\left(\mathrm{I}_{1 k}^{r} \cdot \mathrm{I}_{1 k}^{i}\right)\right\}$,

$\mu_{2(i)}=\max \left\{\left\|g_{2 k}\right\| /\left(\mathrm{I}_{2 k}^{r} \cdot \mathrm{I}_{2 k}^{i}\right)\right\}$

if $\mu_{1(i)} \geq \mu_{2(i)} \&\left|\mathcal{S}_{1}\right|<M_{1}$ then

$\pi_{(i)}=\arg \mu_{1(i)} ; \mathcal{S}_{1}=\mathcal{S}_{1} \cup\left\{\pi_{(i)}\right\} ;$

else

$$
\mathbf{g}_{1(i)}=\mathbf{h}_{\pi_{(i)}} \text {; }
$$

$$
\begin{aligned}
& \pi_{(i)}=\arg \mu_{2(i)} ; \mathcal{S}_{2}=\mathcal{S}_{2} \cup\left\{\pi_{(i)}\right\} \\
& \mathbf{g}_{2(i)}=\mathbf{h}_{\pi_{(i)}} ;
\end{aligned}
$$

end

$i=i+1$;

$\mathcal{T}_{(i)}=\mathcal{T}_{(i-1)}-\left\{\pi_{(i-1)}\right\} ;$

end

Algorithm 1: Semiorthogonal Interference aware User Allocation algorithm (SIUA)

[5] T. Yoo and A. Goldsmith, "On the optimality of multi-antenna broadcast scheduling using zero-forcing beamforming,", IEEE J. Select. Areas Commun., vol. 24, Mar. 2006.

[6] _ "Optimality of zero-forcing beamforming with multiuser diversity," in IEEE ICC, Int. Conf. on Commun., vol. 1, May 2005, pp. 542546.

[7] B. L. Ng, J. Evans, S. Hanly, and D. Aktas, "Distributed downlink beamforming with cooperative base stations," IEEE Trans. Inf. Theory, vol. 54, no. 12, pp. $5491-5499$, dec. 2008.

[8] H. Weingarten, Y. Steinberg, and S. Shamai, "The capacity region of the Gaussian multiple-input multiple-output broadcast channel," IEEE Trans. Inf. Theory, vol. 52, no. 9, pp. 3936-3964, 2006.

[9] M. Diaz, N. Courville, C. Mosquera, G. Liva, and G. Corazza, "Nonlinear interference mitigation for broadband multimedia satellite systems," in Proc. Int. Work. Sat. Space Commun. (IWSSC), Sept. 2007, pp. 61-65.

[10] H. Viswanathan, S. Venkatesan, and H. Huang, "Downlink capacity

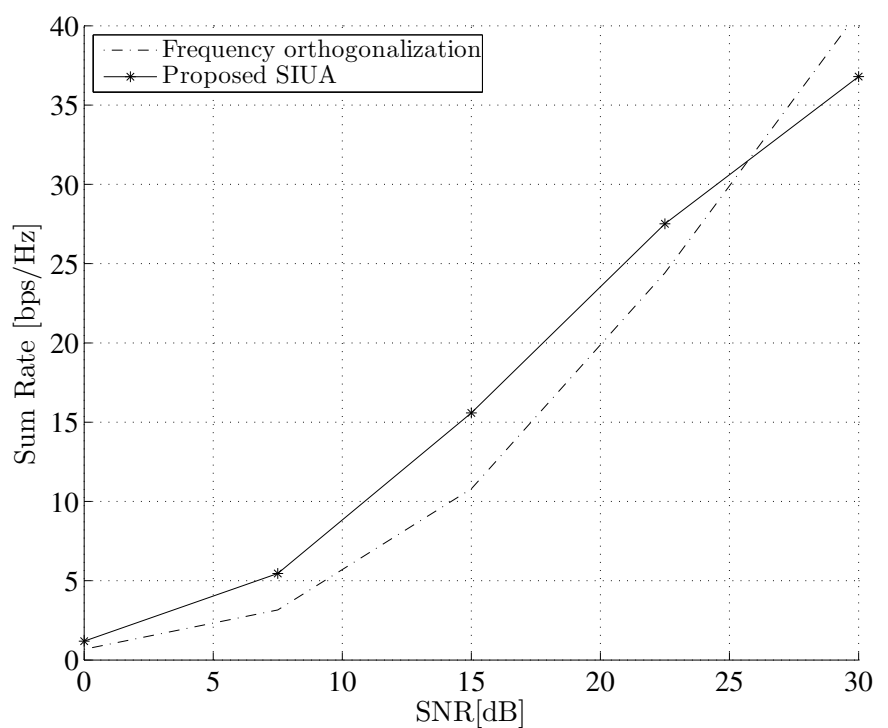

Fig. 3. Comparison of a coordinated system employing SIUA, with a conventional frequency orthogonalization system.

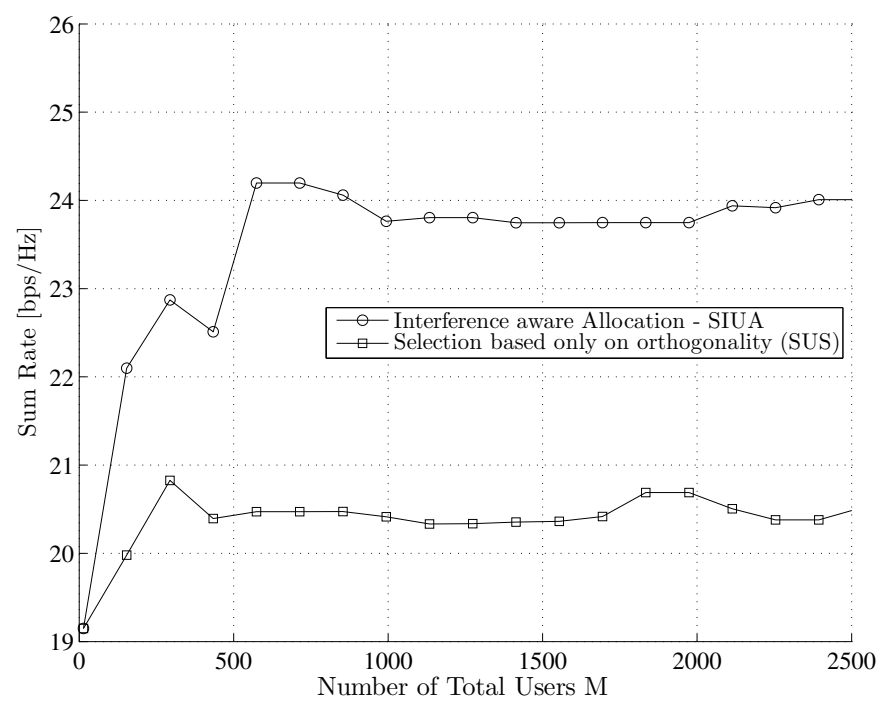

Fig. 4. Algorithm performance with respect to the number of available for selection users.

evaluation of cellular networks with known-interference cancellation," IEEE J. Select. Areas Commun., vol. 21, no. 5, pp. 802-811, 2003.

[11] G. Caire and S. Shamai, "On the achievable throughput of a multiantenna Gaussian broadcast channel," IEEE Trans. Inf. Theory, vol. 49, no. 7, pp. 1691-1706, July 2003.

[12] K. Karakayali, R. Yates, G. Foschini, and R. Valenzuela, "Optimum zero-forcing beamforming with per-antenna power constraints," in IEEE ISIT, Int. Symp. on Inf. Theory, 2008, pp. 101-105.

[13] W. Yu and T. Lan, "Transmitter optimization for the multi-antenna downlink with per-antenna power constraints," IEEE Trans. Signal Process., vol. 55, no. 6, pp. 2646-2660, June 2007.

[14] S. Boyd and L. Vandenberghe, Convex optimization. Cambridge Univ. Press, 2004

[15] S. K. Sharma, S. Chatzinotas, and B. Ottersten, "Exploiting polarization for spectrum sensing in cognitive satcoms," in Proc. 7th Int. Conf. CROWNCOM, Jun. 2012. 